the intestinal ulcerations met with in amobic dysentery are described. The ulcers vary in size from a big pin's head to an area of several square inches, and consist generally of raised yellowish gelatinous patches marked off sharply by a narrow zone of intense congestion from the surrounding healthy mucous membrane. The raised character of the ulcers and the absence of thickening and congestion of the non-ulcerated portions of the bowel distinguish this form of ulceration from that met with in ordinary cases. The largest ulcers are met with in the cæcum and the ascending colon, but the disease never in this variety extends above the iliocreal valve. The liver abscess, even when small, is rarely situated deep in its substance ; it is found just under the surface in the neighbourhood of the suspensory ligament. Great stress is laid on the value of leucocytosis as a diagnostic feature in liver abscess, especially in helping to distinguish it from some forms of malaria. The introduction of concentrated solutions of quinine, 1 in 100 or 1 in 80 , into the incised or aspirated abscess cavity is recommended. Outside the body the amœbæ are rapidly killed by such solutions.

2 Brit. Med. Jour., Sept. 20.

\title{
Diseases of the Heart and Circulation.
}

\author{
(Concluded trom page 197.)
}

Inequality of the Pupils in Aortic Aneurysm.Wall and Walker ${ }^{5}$ have recently offered a new explanation of this well-known fact, which has hitherto been thought to be due to interference with the sympathetic. They state that this theory is unsatisfactory : 1, on anatomical grounds, because in the majority of cases there is no evidence of implication of that portion of the sympathetic nerve trunk containing pupil dilator fibres, and because it is not established that sympathetic filaments supply the sac wall of an aneurysm ; 2 , on physiological grounds ; because the explanation supposes that the same conditions may produce sometimes irritation and sometimes paralysis of the nerve; because cases are rare in which there is evidence apart from the pupil. lary change that the sympathetic is involved; and because the pupillary changes are not those met with in cases where the sympathetic is involved. Alterations in vascular conditions may be associated with alterations in the size of the pupils ; thus high arterial tension is associated with small pupils, and low arterial tension with large ones. The cause of this is probably to be found in the spiral structure of the vessels of the iris. Local inequalities of blood pressure may therefore be associated with inequalities of the pupils ; thus, enlargement of the pupil is frequently associated with diminution of the temporal and radial pulses on the same side of the body; and obstruction of the carotid artery on one side of the neck is associated with enlargement of the pupil on the same side. Similarly digital compression of the carotid artery will cause enlargement of the pupil on the same side. The authors conclude that inequality of pupils associated with thoracic aneurysm is usually due to inequality of blood pressure in the ophthalmic arteries resulting from the abnormal vascular conditions.

Intermittent Claudication.-Walton and Paul 6 suggest the name angina cruris for this disease, which was first described by Charcot in 1858, for the essential symptom is intense paroxysmal pain, whilst the lameness is by no means a constant feature. The painful paroxysms are probably of vascular origin, and result from vascular spasm, perhaps with increased blood-pressure acting on vessels partly occluded whether from local or general disease (aneurysm, syphilis), from senile changes (atheroma), or from congenital tendency to angiofibrosis. The occurrence of the paroxysmal pains with pulseless pedal arteries is too constant to be explained by coincidence, though pulseless arteries may be found without the pains, and conversely The authors think that many cases of recurring local cramps properly belong to this condition, particularly when associated with vascular changes.

Sclerosis of the Superficial Veins.-Sonques and Jauvier 7 have described a case of this very rare condition. A man, aged 40, who had not suffered from syphilis, but was addicted to alcohol, came under observation for incipient pulmonary tuberculosis. The superficial veins of the limbs were indurated, smaller than normal, hard and resistant, and rolled under the fingers like a cord. The induration had come on gradually and the patient was not aware of its existence, nor was he affected thereby. The radial arteries were hard, but the temporal arteries were not tortuous. The heart and kidneys were normal. The lesions differ from those of chronic phlebitis, the indurated vein is like a contracted artery, its lumen is scalloped and narrowed, and the folds formed by the intima are free from clots. Its pathology is obscure ; some have suggested that it is similar to that of arterio sclerosis.

Caisson Disease.-Macmorran ${ }^{8}$ has reported the cases of caisson disease which occurred during the contruction of the Greenwich footway tunnel, which occupied about thirteen months. During the whole of that time there were but nine cases. This small number was due to the ample supply of fresh air. Three of the cases were serious, the others slight, all of them had giddiness as a symptom, only one had pains in the joints, two had pains in the back and thighs, one had recurrent epistaxis, with pain in the nape of the head and forehead, and shortness of breath. It was found that the presence of carbonic acid gas in the air in undue proportions causes an increase in the number of cases of caisson disease. This suggests that under the increased pressure the interchange of gases in the lungs is interfered with, so that the $\mathrm{CO}_{2}$ is less readily eliminated. To this we must add the hyperæmia of the deeper tissues due to the pressure, so that the circulation is retarded. Owing to these two causes, hyperæmia of the nerve centres and accumulated impurities in the blood, there is a double effect on the sympathetic and vasomotor systems, the consequences of which are not manifest till varying times after the cause has been removed, when reaction occurs manifested in the sensory and sympathethic nerves throughout the body. By far the best treatment is the medical lock, into which a purified air should be pumped till the pain goes, and then the pressure very gradually reduced. The air 
pumped in should be purified by passing it over an apparatus containing caustic soda in screens, so that most of the $\mathrm{CO}_{2}$ is removed. Similarly the prevention of the worst form of caisson disease can be prevented by getting rid of the $\mathrm{CO}_{2}$ where it collects in largest quantity, namely, in the shield where the men are working. This was done in the Greenwich tunnel, with the excellent results mentioned above. All men employed should be constantly examined, and should a booming sound in the heart or reduplication of the second sound be heard the man should be suspended altogether.

5 Lancet, July 12. 6 Boston Med. and Surg. Jour., April 3. 7. Lancet, June 28.8 Brit. Med. Jour., April 26.

\section{DiSEASES OF THE ABDOMEN.}

The existence of a "quiescent period" in grave abdominal conditions has been recognised before; but Tubby $^{5}$ gives some practical points in the diagnosis of such conditions. The difficulty is to know if the improvement in condition is only apparent or real. A patient who has shown alarming symptoms gets apparently much better; these symptoms may, however, be only in abeyance, and a worse condition brewing. This quiescent period is a prominent feature in

(1) Injury to intestines, with or without rupture.

(2) Perforation of intestine by ulceration.

(3) Injury to, and bruising of, the peritoneal connections of the viscera, with interference with their blood supply.

(4) Acute disease of genital organs, especially Fallopian tubes.

(5) Acute appendicitis, either ulcerative, perforative, or gangrenous. It is in this disease that the condition is most often met with.

The clinical signs of moment in these doubtful eases are these :-

(1) Repeated records of pulse and temperature may show a very rapid pulse, while the temperature (surface) is abnormal. This is of grave import, and means septic absorption, butit must be observed early in order to prevent saturation with septic products, in order for operation to be of use.

After an acute attack of appendicitis, when the temperature and pulse rate have been high, if a fall occurs and is followed by a rise in a few days, pus, in quantity, is indicated.

(2) A transient or persistent rigidity is most valuable in the absence of other symptoms, and if peristalsis is absent it is convincing as an indication for immediate interference.

(3) Distension lasting longer than 24 to 36 hours in abdominal conditions is of grave moment and must be relieved.

(4) Vomiting occurring in such a quiescent period in spite of good nursing, careful feeding, and sedatives, must not be underestimated.

(5) Leucocytosis : Counts of over 20,000 in cases of appendicitis indicate pus, gangrene, or peritonitis. Below this number nothing is certain, as in 35 per cent. of cases of appendicitis there is a small increase in leucocytes.

Leucocytosis is absent in typhoid, but present in ovarian abscess, perinephritic suppuration, and, indeed, in suppuration generally. The presence of leucocytosis in cases of gastric ulcer, apart from a certain amount due to severe hæmorrhage or digestion, is an indication of perforation or other grave condition.

In this connection Savill ${ }^{6}$ considers that in any grave abdominal trouble the blood count is of the utmost importance, but the presence of pus is to be inferred not by the actual number of the leucocytes as a whole, but from the preponderance over the normal 65 per cent. of the polynuclear cells.

(6) Chest complications persisting when the abdominal symptoms have apparently cleared up indicate exploratory operation for pus in the region of the diaphragm.

In a case giving the symptoms of acute intestinal obstruction, under the care of Tyson and Linington ${ }^{7}$ the cause was found to be embolism of branches of the superior mesenteric artery. This had led to gangrene in a large portion of the bowel and the mesentery right up to the attachment. Extensive removal was not sufficient to save the patient's life, death occurring 36 hours later. They remind us that hæmorrhage from the bowel may be due to this cause ; extreme foetor, with tension and tympanitic swelling of the abdomen show gangrenous bowel and peritonitis. Provided operation is performed early enough the prognosis is not necessarily fatal. Kneading the abdomen is the worst treatment that can befall the case ; opium masks the symptoms. Should the collateral circulation be established, such conditions may subside by themselves, possibly undiagnosed.

From statistics of Massachusetts General Hospital Shattuck ${ }^{8}$ gives the mortality of tubercular peritonitis as $13 \cdot 2$ per cent. This is based upon a series of 98 cases at discharge. The mortality after 2 to 11 years rises to $47 \cdot 3$ per cent. ; ultimate mortality under medical treatment, 68 per cent. ; under surgical treatment, 37.5 per cent. ; two patients have shown recurrence and are now well. The writer concludes from his experience that :-

(1) Patients may recover completely even if the disease be complicated by tubercular disease elsewhere under $(a)$ purely medical treatment; $(b)$ tapping ; $(c)$ incision.

2. Best hygienic surroundings are necessary as in other conditions of tubercular disease.

3 . We are warranted in trying medical treatment for a time, tapping if necessary, for the relief of discomfort.

4. If no improvement or if loss of ground occur in one month or six weeks of medical treatment, then surgery should be advised.

Bernard ${ }^{9}$ draws attention to the resemblance of some cases of lead colic to appendicitis. The diagnosis should be clear, however, in 24 hours. Theodore Fisher ${ }^{10}$ gives as his impression that more cases of appendicitis are met with in the post-mortem room, since operative treatment has become more common, than before.

${ }^{5}$ Lancet, May 10. ${ }^{6}$ Ibid., 7 Ibid, May 3. 8 Amer. Jour. Med. Sci., July 1902. ${ }^{9}$ Brit. Med. Jour., March $1 .{ }^{10}$ Lancet, July 19.

(To be continued.) 